\title{
HYPONORMAL OPERATORS ON UNIFORMLY SMOOTH SPACES
}

\author{
MUNEO CHŌ
}

(Received 30 July 1989)

Communicated by A. J. Pryde

Dedicated to Professor Satoshi Koto in celebration of his having been honoured as an emeritus Professor of Joetsu University of Education

\begin{abstract}
In this paper we will characterize the spectrum of a hyponormal operator and the joint spectrum of a doubly commuting $n$-tuple of strongly hyponormal operators on a uniformly smooth space. We also describe some applications of these results.

1980 Mathematics subject classification (Amer. Math. Soc.) (1985 Revision): 47 A 12.
\end{abstract}

\section{Introduction}

Let $X$ be a complex Banach space. We denote by $X^{*}$ the dual space of $X$ and by $B(X)$ the space of all bounded linear operators on $X$. When $x \in X$ with $\|x\|=1$, we put $D(x)=\left\{f \in X^{*}:\|f\|=f(x)=1\right\}$. Let us set

$$
\pi=\left\{(x, f) \in X \times X^{*}:\|f\|=f(x)=\|x\|=1\right\} .
$$

The numerical range $V(T)$ of $T \in B(X)$ is defined by

$$
V(T)=\{f(T x):(x, f) \in \pi\} .
$$

If $V(T) \subset \mathbb{R}$, then $T$ is called hermitian. An operator $T \in B(X)$ is called hyponormal if there are hermitian operators $H$ and $K$ such that $T=H+i K$ and $C=i(H K-K H) \geq 0$, meaning that $V(C) \subset \mathbb{R}^{+}=\{a \in \mathbb{R}: a \geq 0\}$. A Hyponormal operator $T=H+i K$ is called strongly hyponormal if $H^{2}$ and

(C) 1991 Australian Mathematical Society 0263-6115/91 \$A2.00+0.00 
$K^{2}$ are hermitian. If $T$ is (strongly) hyponormal, then so is $T-\lambda$ for every $\lambda \in \mathbb{C}$. For an operator $T=H+i K$, we denote the operator $H-i K$ by $\bar{T}$.

REMARK. There is an hermitian operator $H$ such that $H^{2}$ is not hermitian. However, if $H$ is hermitian, then

$$
V\left(H^{2}\right) \subset\{z \in \mathbb{C}: \operatorname{Re} z \geq 0\} .
$$

Hence, if $T$ is a strongly hyponormal operator, then

$$
V(\bar{T} T) \subset \mathbb{R}^{+} .
$$

For an operator $T \in B(X)$, the spectrum, the approximate point spectrum, the point spectrum, the kernel and the dual operator of $T$ are denoted by $\sigma(T), \sigma_{\pi}(T), \sigma_{p}(T), \operatorname{Ker}(T)$ and $T^{*}$, respectively. The following facts are well-known:

(1) $\operatorname{co} \sigma(T) \subset \overline{V(T)}$, where co $E$ and $\bar{E}$ are the convex hull and the closure of $E$, respectively;

(2) $V(T) \subset V\left(T^{*}\right) \subset \overline{V(T)}$.

Hence if $T$ is hermitian and positive, then $T^{*}$ is hermitian and positive, respectively. And if $T$ is (strongly) hyponormal, then so is $\bar{T}^{*}$. We set, for $t>0$,

$$
\rho(t)=\sup \left\{\frac{1}{2}(\|x+y\|+\|x-y\|)-1:\|x\|=1,\|y\| \leq t\right\} .
$$

A Banach space $X$ is called uniformly smooth if

$$
\frac{\rho(t)}{t} \rightarrow 0 \text { as } t \rightarrow 0 \text {. }
$$

A Banach space $X$ is called smooth if the set $D(x)$ is a singleton for each $x \in X$ with $\|x\|=1$. The following facts are well-known:

(1) $X$ is uniformly smooth if and only if $X^{*}$ is uniformly convex;

(2) if $X$ is uniformly smooth, then $X$ is smooth.

See Beauzamy [3] for details.

\section{The spectrum of a hyponormal operator}

LemMA 1. Let $X$ be uniformly smooth. Let $T=H+i K$ be a hyponormal operator on $X$. If $\bar{T} T$ is not invertible, then $T \bar{T}$ is not invertible.

Proof. Let $C=i(H K-K H) \geq 0$. Since then $(\bar{T} T)^{*}=H^{* 2}+K^{* 2}+C^{*}$ is not invertible and 0 belongs to the boundary of $\sigma\left((\bar{T} T)^{*}\right)$, there exists a sequence $\left\{f_{n}\right\}$ of unit vectors in $X^{*}$ such that

$$
\left(H^{* 2}+K^{* 2}\right) f_{n}+C^{*} f_{n} \rightarrow 0 \text {. }
$$


Choose a sequence $\left\{x_{n}\right\}$ of unit vectors in $X$ such that $\left(x_{n}, f_{n}\right) \in \pi$. Since then $\operatorname{Re} \hat{x}_{n}\left(\left(H^{* 2}+K^{* 2}\right) f_{n}\right) \geq 0, \hat{x}_{n}\left(C^{*} f_{n}\right) \geq 0$ and $X^{*}$ is uniformly convex, by [16, Theorem 2.5] it follows that $C^{*} f_{n} \rightarrow 0$. Therefore we have

$$
\left(H^{* 2}+K^{* 2}\right) f_{n}-C^{*} f_{n} \rightarrow 0 .
$$

Hence $(T \bar{T})^{*}=H^{* 2}+K^{* 2}-C^{*}$ is not invertible and therefore $T \bar{T}$ is not invertible either.

THeOREM 2. Let $X$ be uniformly smooth. Let $T=H+i K$ be a hyponormal operator on $X$. Then $\sigma(T)=\sigma_{\pi}\left(T^{*}\right)$.

Proof. It is clear that $\sigma_{\pi}\left(T^{*}\right) \subset \sigma(T)$. Since $T-\lambda$ is hyponormal for every $\lambda \in \mathbb{C}$, we need only prove that if $0 \in \sigma(T)$, then $0 \in \sigma_{\pi}\left(T^{*}\right)$. Hence by Lemma 1 we may assume that $T \bar{T}$ is not invertible. Then there exists a sequence $\left\{f_{n}\right\}$ of unit vecotrs in $X^{*}$ such that $\bar{T}^{*} T^{*} f_{n} \rightarrow 0$. Since $X^{*}$ is uniformly convex and $\bar{T}^{*}$ is a hyponormal operator on $X$, by [16, Theorem 2.7] it follows that $H^{*} T^{*} f_{n} \rightarrow 0$ and $K^{*} T^{*} f_{n} \rightarrow 0$. Hence we have $T^{* 2} f_{n} \rightarrow 0$. By the spectral mapping theorem for the approximate point spectrum it follows that $0 \in \sigma_{\pi}\left(T^{*}\right)$.

THEOREM 3. Let $X$ be uniformly smooth. Let $T=H+i K$ be a strongly hyponormal operator on $X$. If $a+i b \in \sigma(T)$, then $a \in \sigma(H)$ and $b \in \sigma(K)$.

Proof. Since $T-\lambda$ is hyponormal for every $\lambda \in \mathbb{C}$, we need only prove that if $0 \in \sigma(T)$ then $0 \in \sigma(H)$. There exists $\alpha \in \mathbb{R}$ such that $0+i \alpha$ is in the boundary of $\sigma(T)$. Hence there exists a sequence $\left\{x_{n}\right\}$ of unit vectors in $X$ such that $(T-i \alpha) x_{n} \rightarrow 0$. Therefore, we have

$$
\overline{(T-i \alpha)} \cdot(T-i \alpha) x_{n}=\left(H^{2}+(K-\alpha)^{2}+C\right) x_{n} \rightarrow 0,
$$

where $c=i(H K-K H) \geq 0$. Since $T$ is strongly hyponormal, $H^{2}+$ $(K-\alpha)^{2}+C$ is hermitian. By [15, Theorem 3.11], it follows that

$$
\left(H^{2 *}+(K-\alpha)^{2 *}+C^{*}\right) f_{n} \rightarrow 0,
$$

where $f_{n} \in D\left(x_{n}\right)$. Since $X^{*}$ is uniformly convex and $H^{2 *},(K-\alpha)^{2 *}$ and $C^{*}$ are all positive, we have $H^{2 *} f_{n} \rightarrow 0$. Hence we have $0 \in \sigma(H)$.

Next since $i T=K+i(-H)$ is strongly hyponormal and $b-i a \in \sigma(-i T)$, that $b \in \sigma(K)$ can be proved analogously. 
Corollary 4. Let $X$ be uniformly smooth. Let $T=H+i K$ be a strongly hyponormal operator on $X$. Then $\operatorname{Re} \sigma(T)=\sigma(H)$ and $\operatorname{Im} \sigma(T)=\sigma(K)$.

A proof follows easily from Theorem 3 above and [9, Theorem 1].

\section{The joint spectrum for strongly hyponormal operators}

Let $\mathbf{T}=\left(T_{1}, \ldots, T_{n}\right)$ be a commuting $n$-tuple of operators on a Banach space $X$. We denote the (Taylor) joint spectrum of $T$ by $\sigma(T)$. We refer the reader to Taylor [20] for the definition of $\sigma(T)$. For a commuting $n$-tuple $\mathbf{T}=\left(T_{1}, \ldots, T_{n}\right)$ of operators, the joint approximate point spectrum and the joint point spectrum of $T$ are denoted by $\sigma_{\pi}(T)$ and $\sigma_{p}(T)$, respectively.

For a commuting $n$-tuple $\mathrm{T}=\left(T_{1}, \ldots, T_{n}\right)$ such that $T_{j}=H_{j}+i K_{j}$ $(j=1, \ldots, n)$, a point $\mathbf{z}=\left(z_{1}, \ldots, z_{n}\right) \in \mathbb{C}^{n}$ is in the complete star spectrum $\sigma_{c s}(\mathbf{T})$ of $\mathbf{T}$ if there is some partition $\left\{j_{1}, \ldots, j_{k}\right\} \cup\left\{l_{1}, \ldots, l_{m}\right\}$ $=\{1, \ldots, n\}$ such that

$$
\sum_{\mu=1}^{k} \overline{\left(T_{j_{\mu}}-z_{j_{\mu}}\right)}\left(T_{j_{\mu}}-z_{j_{\mu}}\right)+\sum_{v=1}^{m}\left(T_{l_{v}}-z_{l_{v}}\right) \overline{\left(T_{l_{v}}-z_{l_{v}}\right)}
$$

is not invertible.

For a commuting $n$-tuple $\mathbf{T}=\left(T_{1}, \ldots, T_{n}\right)$ of hyponormal operators, $\mathbf{T}$ is called a doubly commuting $n$-tuple if $T_{i} \bar{T}_{j}=\bar{T}_{j} T_{i}$ for every $i \neq j$. It is easy to see that $\mathrm{T}$ is a doubly commuting $n$-tuple if and only if $H_{i}$ and $K_{i}$ commute with $H_{j}$ and $K_{j}$ for every $i \neq j$. In [10], we showed the following theorem. The assumption of the uniform convexity in the theorem is not needed.

Theorem A [10, Theorem 5]. Let $\mathbf{T}=\left(T_{1}, \ldots, T_{n}\right)$ be a doubly commuting n-tuple of hyponormal operators on $X$. Then $\sigma(\mathbf{T}) \subset \sigma_{c s}(\mathbf{T})$.

LemMA 5. Let $X$ be uniformly smooth. Let $\mathbf{T}=\left(T_{1}, \ldots, T_{n}\right)$ be a doubly commuting $n$-tuple of strongly hyponormal operators on $X$. If $\sum_{i=1}^{k} \bar{T}_{i} T_{i}+$ $\sum_{i=k+1}^{n} T_{i} \bar{T}_{i}$ is not invertible, then $\sum_{i=1}^{n} T_{i} \bar{T}_{i}$ is not invertible.

Proof. By the assumption, $\sum_{i=1}^{k} T_{i}^{*} \bar{T}_{i}^{*}+\sum_{i=k+1}^{n} \bar{T}_{i}^{*} T_{i}^{*}$ is not invertible. Let $\mathbf{S}=\left(\bar{T}_{1} T_{1}, \ldots, \bar{T}_{k} T_{k}, T_{k+1} \bar{T}_{k+1}, \ldots, T_{n} \bar{T}_{n}\right)$. Then $\mathbf{S}$ is a commuting $n$-tuple of operators with positive spectra. By the spectral mapping theorem for the joint approximate point spectrum, it follows that there exists $\left(\alpha_{1}, \ldots, \alpha_{n}\right) \in \sigma_{\pi}\left(\mathbf{S}^{*}\right)$ such that $\alpha_{1}+\cdots+\alpha_{n}=0$, where 
$\mathrm{S}^{*}=\left(T_{1}^{*} \bar{T}_{1}^{*}, \ldots, T_{k}^{*} \bar{T}_{k}^{*}, \bar{T}_{k+1}^{*} T_{k+1}^{*}, \ldots, \bar{T}_{n}^{*} T_{n}^{*}\right)$. Since $T_{i}$ is strongly hyponormal, it follows that $\alpha_{i} \geq 0$, for $i=1,2, \ldots, n$. Therefore we have $(0, \ldots, 0) \in \sigma_{\pi}\left(\mathbf{S}^{*}\right)$. Hence there exists a sequence $\left\{f_{j}\right\}$ of unit vectors in $X^{*}$ such that

$T_{i}^{*} \bar{T}_{i}^{*} f_{j} \rightarrow 0 \quad$ and $\quad \bar{T}_{l}^{*} T_{l}^{*} f_{j} \rightarrow 0 \quad$ for $i=1, \ldots, k$ and $l=k+1, \ldots, n$. Let $C_{i}=i\left(H_{i} K_{k}-K_{i} H_{i}\right) \geq 0$ for $i=1, \ldots, k$. Then since

$$
\left(H_{i}^{* 2}+K_{i}^{* 2}\right) f_{j}+C_{i}^{*} f_{j} \rightarrow 0
$$

and $X^{*}$ is uniformly convex, by the method of the proof of Lemma 1 we have that $C_{i}^{*} f_{j} \rightarrow 0$, for $i=1, \ldots, k$. Hence we have that

$$
\bar{T}_{i}^{*} T_{i}^{*} f_{j} \rightarrow 0 \text { for } i=1, \ldots, n .
$$

Therefore, $\sum_{i=1}^{n} T_{k} \bar{T}_{i}$ is not invertible.

THEOREM 6. Let $X$ be uniformly smooth. Let $\mathbf{T}=\left(T_{1}, \ldots, T_{n}\right)$ be a doubly commuting $n$-tuple of strongly hyponormal operators on $X$.

Then

$$
\sigma(\mathbf{T})=\sigma_{\pi}\left(\mathbf{T}^{*}\right),
$$

where $\mathbf{T}^{*}=\left(T_{1}^{*}, \ldots, T_{n}^{*}\right)$.

Proof. Since $\sigma(\mathbf{T})=\sigma\left(\mathbf{T}^{*}\right)$, it is clear that

$$
\sigma_{\pi}\left(\mathbf{T}^{*}\right) \subset \sigma(\mathbf{T}) .
$$

By using Lemma 5 and Theorem A, we may assume that $\sum_{i=1}^{n} T_{i} \bar{T}_{i}$ is not invertible. Hence we have

$$
0 \in \sigma\left(\left(\sum_{i=1}^{n} T_{i} \bar{T}_{i}\right)^{*}\right)=\sigma\left(\sum_{i=1}^{n} \bar{T}_{i}^{*} T_{i}^{*}\right) .
$$

Since 0 is in the boundary of $\sigma\left(\sum_{i=1}^{n} \bar{T}_{i}^{*} T_{i}^{*}\right)$, by the proof of Lemma 5 there exists a sequence $\left\{f_{k}\right\}$ of unit vectors in $X^{*}$ such that

$$
\bar{T}_{i}^{*} T_{i}^{*} f_{k} \rightarrow 0 \text { for } i=1,2, \ldots, n .
$$

Since $X^{*}$ is uniformly convex and every $\bar{T}_{i}^{*}$ is a hyponormal operator on $X^{*}$, we have

$$
\left(T_{i}^{*}\right)^{2} f_{k} \rightarrow 0 \quad(i=1,2, \ldots, n) .
$$

Hence we have $0 \in \sigma_{\pi}\left(\mathbf{T}^{* 2}\right)$, where $\mathbf{T}^{* 2}=\left(T_{1}^{* 2}, \ldots, T_{n}^{* 2}\right)$. By the spectral mapping theorem for the joint approximate point spectrum, it follows that

$$
0 \in \sigma_{\pi}\left(\mathbf{T}^{*}\right) \text {. }
$$


Since $\mathbf{T}-\mathbf{z}=\left(T_{1}-z_{1}, \ldots, T_{n}-z_{n}\right)$ is a doubly commuting $n$-tuple of strongly hyponormal operators, Theorem $A$ and Lemma 5 imply that $\sigma(\mathbf{T}) \subset$ $\sigma_{c s}(\mathbf{T}) \subset \sigma_{\pi}\left(\mathbf{T}^{*}\right)$. This complets the proof.

\section{Applications}

In the following we shall represent a construction of de Barra ([1] and [2]) embedding a Banach space in a larger space $X^{0}$. Then the mapping $T \rightarrow T^{0}$ is an isometric isomorphism of $B(X)$ onto a closed subalgebra of $B\left(X^{0}\right)$. Let Lim be a fixed Banach limit on the space of all bounded sequences of complex numbers with the norm $\left\|\left\{\lambda_{n}\right\}\right\|=\sup \left\{\left|\lambda_{n}\right|: n \in \mathbb{N}\right\}$. Let $\tilde{X}$ be the space of all bounded sequences $\left\{x_{n}\right\}$ of $X$. Let $N$ be the subspace of $\tilde{X}$ consisting of all bounded sequences $\left\{x_{n}\right\}$ with $\operatorname{Lim}\left\|x_{n}\right\|^{2}=0$. The space $X^{0}$ is defined as the completion of the quotient space $\tilde{X} / N$ with respect to the norm $\left\|\left\{x_{n}\right\}+N\right\|=\left(\operatorname{Lim}\left\|x_{n}\right\|^{2}\right)^{1 / 2}$. Then the following results hold for $T \in B(X)$ :

$$
\sigma(T)=\sigma\left(T^{0}\right), \sigma_{\pi}(T)=\sigma_{\pi}\left(T^{0}\right)=\sigma_{p}\left(T^{0}\right) \quad \text { and } \quad \overline{\operatorname{co}} V(T)=V\left(T^{0}\right) .
$$

Hence, if $T$ is (strongly) hyponormal, then so is $T^{0}$. Moreover, it follows for $\mathbf{T}=\left(T_{1}, \ldots, T_{n}\right)$ that $\sigma_{\pi}(\mathbf{T})=\sigma_{p}\left(\mathbf{T}^{0}\right)$, where $\mathbf{T}^{0}=\left(T_{1}^{0}, \ldots, T_{n}^{0}\right)$

In this section we shall need the following result.

THEOREM B [2, THEOREM 2.7]. $X$ is uniformly convex if and only if $X^{0}$ is uniformly convex.

THEOREM 7. Let $X$ be uniformly smooth. Let $\mathbf{T}=T_{1}, \ldots, T_{n}$ ) be a doubly commuting $n$-tuple of strongly hyponormal operators on $X$ such that $T_{j}=H+i K_{j}(j=1, \ldots, n)$. If $\left(\lambda_{1}+i \mu_{1}, \ldots, \lambda_{n}+i \mu_{n}\right) \in \sigma(\mathbf{T})$, then $\left(\lambda_{1}, \ldots, \lambda_{n}\right) \in \sigma(\mathbf{H})$ and $\left(\mu_{1}, \ldots, \mu_{n}\right) \in \sigma(\mathbf{K})$, where $\mathbf{H}=\left(H_{1}, \ldots, H_{n}\right)$ and $\mathbf{K}=\left(K_{1}, \ldots, K_{n}\right)$.

Proof. First, we shall prove that if $0 \in \sigma(\mathbf{T})$, then $0 \in \sigma(\mathbf{H})$, by the method of induction. For $n=1$, it is true from Theorem 3. We assume that the theorem holds for such $(n-1)$-tuples. Since $0 \in \sigma(T)$, Theorem 6 implies that $0 \in \sigma_{\pi}\left(\mathbf{T}^{*}\right)$, where $\mathbf{T}^{*}=\left(T_{1}^{*}, \ldots, T_{n}^{*}\right)$. Consider the larger space $X^{* 0}$ of $X^{*}$ and the representation $T \rightarrow T^{0}$ in the sense of de Barra. Then $X^{* 0}$ is uniformly convex and $0 \in \sigma_{p}\left(\mathrm{~T}^{* 0}\right)$, where $\mathrm{T}^{* 0}=\left(T_{1}^{* 0}, \ldots, T_{n}^{* 0}\right)$.

Let $Y=\left\{f \in X^{* 0}: T_{n}^{* 0} f=0\right\}$. Then $Y$ is a non-zero (uniformly convex) subspace of $X^{* 0}$ and there exists a non-zero vector $g$ in $Y$ such 
that $T_{j}^{* 0} g=0 \quad(j=1, \ldots, n-1)$. Since $\mathbf{T}^{* 0}$ is a doubly commuting $n$-tuple, $Y$ is invariant for all $H_{j}^{* 0}$ and $K_{j}^{* 0}(j=1, \ldots, n-1)$. Let $\mathbf{S}=\left(T_{1 \mid Y}^{* 0}, \ldots, T_{n-1 \mid Y}^{* 0}\right)$. Since then $0 \in \sigma_{p}(\mathbf{S})$, it follows that $0 \in \sigma\left(\mathbf{S}^{*}\right)$, where $\mathbf{S}^{*}=\left(\left(T_{1 \mid Y}^{* 0}\right)^{*}, \ldots,\left(T_{n-1 \mid Y}^{* 0}\right)^{*}\right)$. Since $Y^{*}$ is uniformly smooth and $\mathbf{S}^{*}$ is a doubly commuting $(n-1)$-tuple of strongly hyponormal operators, by the assumption of induction it follows that $0 \in \sigma\left(\mathbf{H}^{\prime *}\right)=\sigma\left(\mathbf{H}^{\prime}\right)$, where $\mathbf{H}^{\prime}=\left(H_{\left.1\right|_{Y}}^{* 0}, \ldots, H_{n-\left.1\right|_{Y}}^{* 0}\right)$. By [6, Theorem 2.1], it follows that

$$
0 \in \sigma\left(\mathbf{H}^{\prime}\right)=\sigma_{\pi}\left(\mathbf{H}^{\prime}\right)=\sigma_{p}\left(\mathbf{H}^{\prime}\right) .
$$

let $Z=\left\{f \in X^{* 0}: H_{j}^{* 0} f=0\right.$ for $\left.j=1, \ldots, n-1\right\}$. Since then $Y \cap Z \supsetneqq$ $\{0\}$ and $Z$ is invariant for $H_{n}^{* 0}$ and $K_{n}^{* 0}$, by the same calculation as above it follows that there exists non-zero vector $h \in Z$ such that $H_{n}^{* 0} h=0$. Hence we have $0 \in \sigma_{p}\left(\mathbf{H}^{* 0}\right)$. Since $\sigma_{p}\left(\mathbf{H}^{* 0}\right)=\sigma_{\pi}\left(\mathbf{H}^{*}\right)=\sigma\left(\mathbf{H}^{*}\right)=\sigma(\mathbf{H})$, we have $0 \in \sigma(\mathbf{H})$. Since $\mathbf{T}-\mathbf{Z}=\left(T_{1}-z_{1}, \ldots, T_{n}-z_{n}\right)$ is a doubly commuting $n$-tuple of strongly hyponormal operators for every $z \in \mathbb{C}^{n}$, it holds that if $\left(\lambda_{1}+i \mu_{1}, \ldots, \lambda_{n}+i \mu_{n}\right) \in \sigma(\mathbf{T})$, then $\left(\lambda_{1}, \ldots, \lambda_{n}\right) \in \sigma(\mathbf{H})$.

Next since $-i \mathrm{~T}=\left(-i T_{1}, \ldots,-i T_{n}\right)$ is a double commuting $n$-tuple of strongly hyponormal operators and $\left(\mu_{1}-i \lambda_{1}, \ldots, \mu_{n}-i \lambda_{n}\right) \in \sigma(-i \mathbf{T})$, we see that $\left(\mu_{1}, \ldots, \mu_{n}\right) \in \sigma(\mathbf{K})$ can be proved analogously.

Theorem C [8, Theorem 6]. Let $X$ be uniformly convex. Let $\mathbf{T}=$ $\left(T_{1}, \ldots, T_{n}\right)$ be a doubly commuting $n$-tuple of hyponormal operators on $X$ such that $T_{j}=H_{j}+i K_{j}(j=1, \ldots, n)$. Let $\mathbf{H}=\left(H_{1}, \ldots, H_{n}\right)$ and $\mathbf{K}=\left(K_{1}, \ldots, K_{n}\right)$.If $\left(\lambda_{1}, \ldots, \lambda_{n}\right) \in \sigma(\mathbf{H})$ then there exist $\left(\mu_{1}, \ldots, \mu_{n}\right) \in$ $\mathbb{R}^{n}$ and a sequence $\left\{x_{k}\right\}$ of unit vectors in $X$ such that

$$
\left(H_{j}-\lambda_{j}\right) x_{k} \rightarrow 0 \text { and }\left(K_{j}-\mu_{j}\right) x_{k} \rightarrow 0, \quad j=1, \ldots, n,
$$

that is, $\left(\lambda_{1}+i \mu_{1}, \ldots, \lambda_{n}+i \mu_{n}\right) \in \sigma(\mathbf{T})$.

An analogous result holds for $\sigma(\mathbf{K})$.

TheOREM 8. Let $X$ be uniformly smooth. Let $\mathbf{T}=\left(T_{1}, \ldots, T_{n}\right)$ be a doubly commuting n-tuple of hyponormal operators on $X$ such that $T_{j}=$ $H_{j}+i K_{j}(j=1, \ldots, n)$. Let $\mathbf{H}=\left(H_{1}, \ldots, H_{n}\right)$ and $\mathbf{K}=\left(K_{1}, \ldots, K_{n}\right)$. If $\left(\lambda_{1}, \ldots, \lambda_{n}\right) \in \sigma(\mathbf{H})$ then there exists $\left(\mu_{1}, \ldots, \mu_{n}\right) \in \mathbb{R}^{n}$ such that $\left(\mu_{1}, \ldots\right.$, $\left.\mu_{n}\right) \in \sigma(\mathbf{K})$ and $\left(\lambda_{1}+i \mu_{1}, \ldots, \lambda_{n}+i \mu_{n}\right) \in \sigma(\mathbf{T})$.

An analogous result holds for $\sigma(\mathbf{K})$.

Proof. Since $\mathbf{H}$ is a commuting $n$-tuple of hermitian operators, by [6, Theorem 2.1] it follows that

$$
\sigma(\mathbf{H})=\sigma\left(\mathbf{H}^{*}\right)=\sigma_{\pi}\left(\mathbf{H}^{*}\right) .
$$


Let $\overline{\mathbf{T}}^{*}=\left(T_{1}^{*}, \ldots, \bar{T}_{n}^{*}\right)$. Then $\overline{\mathbf{T}}^{*}$ is a double commuting $n$-tuple of hyponormal operators on the uniformly convex space $X^{*}$. By Theorem $\mathrm{C}$ we have that there exist $\left(\mu_{1}^{\prime}, \ldots, \mu_{n}^{\prime}\right) \in \mathbb{R}^{n}$ and a sequence $\left\{g_{k}\right\}$ of unit vectors in $X^{*}$ such that

$$
\left(H_{j}^{*}-\lambda_{j}\right) g_{k} \rightarrow 0 \text { and }\left(-K_{j}^{*}-\mu_{j}^{\prime}\right) g_{k} \rightarrow 0 \quad \text { for } j=1, \ldots, n \text {. }
$$

Hence let $\mu_{j}=-\mu_{j}^{\prime}(j=1, \ldots, n)$. Then this $\mu=\left(\mu_{1}, \ldots, \mu_{n}\right)$ is an element as required.

The proof for the case of $\sigma(\mathbf{K})$ follows analogously.

Corollary 9. Let $X$ be uniformly smooth. Let $\mathbf{T}=\left(T_{1}, \ldots, T_{n}\right)$ be a doubly commuting $n$-tuple of strongly hyponormal operators on $X$ such that $T_{j}=H_{j}+i K_{j}(j=1, \ldots, n)$. Then $\sigma(\mathbf{H})=\{\operatorname{Rez}: \mathbf{z} \in \sigma(\mathbf{T})\}$ and $\sigma(\mathbf{K})=\{\operatorname{Im} \mathbf{z}: \mathbf{z} \in \sigma(\mathbf{T})\}$, where $\mathbf{H}=\left(H_{1}, \ldots, H_{n}\right), \mathbf{K}=\left(K_{1}, \ldots, K_{n}\right)$, $\operatorname{Re} z=\left(\operatorname{Re} z_{1}, \ldots, \operatorname{Re} z_{n}\right)$ and $\operatorname{Im} z=\left(\operatorname{Im} z_{1}, \ldots, \operatorname{Im} z_{n}\right)$.

A proof follows from Theorems 7 and 8 .

For a commuting $n$-tuple $\mathbf{T}=\left(T_{1}, \ldots, T_{n}\right)$ of operators, the joint numerical range $V(\mathbf{T})$ of $\mathbf{T}$ is defined by

$$
V(\mathbf{T})=\left\{\left(f\left(T_{1} x\right), \ldots, f\left(T_{n} x\right)\right):(x, f) \in \pi\right\} .
$$

Then the following two theorems hold.

Theorem D [19, Corollary 2.3]. Let $\mathbf{T}=\left(T_{1}, \ldots, T_{n}\right)$ be a commuting n-tuple of operators. Then $\operatorname{co} \sigma(\mathbf{T}) \subset \overline{V(\mathbf{T})}$.

THEOREM E [6, THEOREM 2]. Let $X$ be uniformly smooth. Let $T$ be a hyponormal operator on $X$. Then $\operatorname{co} \sigma(T)=\overline{V(T)}$.

THEOREM 10. Let $X$ be uniformly smooth, Let $\mathbf{T}=\left(T_{1}, \ldots, T_{n}\right)$ be a doubly commuting n-typle of hyponormal operators on $X$. Then $\operatorname{co} \sigma(\mathbf{T})=$ $\overline{V(\mathbf{T})}$. Moreover, if $\mathbf{T}=\left(T_{1}, \ldots, T_{n}\right)$ is a doubly commuting $n$-tuple of strongly hyponormal operators on $X$, then $\operatorname{co~} \sigma_{\pi}\left(\mathbf{T}^{*}\right)=\overline{V(\mathbf{T})}$.

Proof. By Theorem D, we can assume that co $\sigma(\mathbf{T}) \nsubseteq \overline{V(T)}$. Suppose that $\left(\alpha_{1}, \ldots, \alpha_{n}\right) \in \overline{V(T)}-\operatorname{co} \sigma(\mathbf{T})$. Then there exists a linear functional $\phi$ on $\mathbb{C}^{n}$ and a real number $r$ such that

$$
\operatorname{Re} \phi(\mathbf{z})<r<\operatorname{Re} \phi(\alpha) \quad(\mathrm{z} \in \operatorname{co} \sigma(\mathbf{T})) .
$$


Let $\phi(\mathbf{z})=t_{1} z_{1}+\cdots+t_{n} z_{n} \quad\left(\mathbf{z}=\left(z_{1}, \ldots, z_{n}\right) \in \mathbb{C}^{n}\right)$. By applying the spectral mapping theorem to the linear functional $\phi$, it follows that

$$
\operatorname{Re} z<r<\operatorname{Re} \phi(\alpha) \quad\left(z \in \sigma\left(\sum_{i=1}^{n} t_{i} T_{i}\right)\right) .
$$

Therefore, we have that

$$
\operatorname{co} \sigma\left(\sum_{i=1}^{n} t_{i} T_{i}\right) \nsubseteq \overline{V\left(\sum_{i=1}^{n} t_{i} T_{i}\right)} .
$$

Since $\sum_{i=1}^{n} t_{i} T_{i}$ is a hyponormal operator, this yields a contradiction to Theorem $\mathbf{E}$.

\section{Acknowledgement}

We would like to express our cordial thanks to the referee for his kind advice.

\section{References}

[1] G. de Barra, 'Some algebras of operators with closed convex numerical ranges', Proc. Roy. Irish Acad. 72 (1972), 149-154.

[2] G. de Barra, 'Generalized limits and uniform convexity', Proc. Roy. Irish Acad. 74 (1974), 73-77.

[3] B. Beauzamy, Introduction to Banach spaces and their geometry, (North-Holland, 1985).

[4] F. F. Bonsall and J. Duncan, Numerical ranges of operators on normed spaces and elements of normed algebras, (Cambridge Univ. Press, 1971).

[5] F. F. Bonsall and J. Duncan, Numerical ranges II, (Cambridge Univ. Press, 1973).

[6] M. Cho, 'Joint spectra of commuting normal operators on Banach spaces', Glasgow Math. J. 30 (1988), 339-345.

[7] M. Chō, 'Hyponormal operators on uniformly convex spaces', Acta Sci. Math. (Szeged), to appear.

[8] M. Cho, 'Joint spectra of commuting pairs and uniform convexity', Rev. Roumaine Math. Pures Appl. 34 (1989), 607-614.

[9] M. Chס, 'Semi-normal operators on uniformly smooth Banach spaces', Glasgow Math. J. 42 (1990), 185-192.

[10] M. Cho 'Joint spectra of strongly hyponormal operators on Banach spaces', J. Math. Soc. Japan 42 (1990), 185-192.

[11] M. Cho,, 'Weyl's theorem for hyponormal operators on Banach spaces', J. Math. Anal. Appl., to appear.

[12] M.-D. Choi and C. Davis, 'The spectral mapping theorem for joint approximate point spectrum', Bull. Amer. Math. Soc. 80 (1974), 317-321.

[13] R. Curto, 'On the connectedness of invertible n-tuples', Indiana Math. J. 29 (1980), 393-406. 
[14] R. Harte, 'The spectral mapping theorem in several variables', Bull. Amer. Math. Soc. 78 (1972), 871-875.

[15] K. Mattila, 'Normal operators and proper boundary points of the spectra of operators on Banach space', Ann. Acad. Sci. Fenn. Ser. A I D Math. Dissertationes 19 (1978).

[16] K. Mattila, 'Complex strict and uniform convexity and hyponormal operators', Math. Proc. Cambridge Philos. Soc. 96 (1986), 484-493.

[17] A. McIntosh, A. J. Pryde and W. Ricker, 'Comparision of the joint spectra of certain classes of commuting operators', Studia Math. 88 (1987), 23-36.

[18] A. McIntosh, A. J. Pryde and W. Ricker, 'Systems of operator equations and pertubation of spectral subspaces of commuting operators', Michigan Math. J. 35 (1988), 43-64.

[19] Z. Slodkowski and W. Zelazko, 'On joint spectra of commuting families of operators', Studia Math. 50 (1974), 127-148.

[20] J. L. Taylor, 'A joint spectrum for several commuting operators', J. Funct. Anal. 6 (1970), 172-191.

[21] J. L. Taylor, 'The analytic functional calculus for several commuting operators', Acta Math. 6 (1970), 1-38.

[22] V. Wrobel, 'Joint spectra and joint numerical ranges for pairwise commuting operators in Banach spaces', Glasgow Math. J. 30 (1988), 145-153.

\author{
Joetsu University of Education \\ Joetsu, Niigata 943 \\ Japan
}

